\title{
Nucleon spin structure functions in the chiral quark model
}

\author{
Chun-Khiang Chua, W-Y.P. Hwang \\ Department of Physics, National Taiwan University, Taipei, 106 Taiwan \\ Center for Theoretical Physics, Laboratory of Nuclear Sciences and Department of Physics, Massachusetts Institute of Technology, \\ Cambridge, MA 02139, USA
}

Received 6 March 1998; received in revised form 8 January 1999

Editor: J.-P. Blaizot

\begin{abstract}
We show that the nucleon spin structure functions, as extracted from the recent experiments carried out by the European Muon Collaboration (EMC), the SLAC E143 and E154 Collaborations, and the Spin Muon Collaboration (SMC), can be understood quantitatively in the chiral quark model, both for the measured first moment $\Gamma_{1}=\int_{0}^{1} g_{1}(x) d x$ and the functional form (or the shape) of the spin structure function $g_{1}(x)$ (as a function of the Bjorken scaling variable $x$ ). The success suggests strongly that the nonperturbative effects due to Goldstone bosons play a significant role, and should be taken into account, in the kinematic range of the momentum transfer squared $Q^{2}$ of several $\mathrm{GeV}^{2}$. (C) 1999 Published by Elsevier Science B.V. All rights reserved.
\end{abstract}

PACS: 13.60.Hb; 14.20.Dh; 11.30.Rd

Recent experiments carried out by the European Muon Collaboration (EMC) [1], the SLAC E143 and E154 Collaborations [2,3], and the Spin Muon Collaboration (SMC) [4] on polarized deep inelastic scattering (DIS) have clarified the picture regarding the nucleon spin structure functions $g_{1}^{p}(x)$ and $g_{1}^{n}(x)$. In particular, the initial finding [1] that the flavor-singlet axial coupling $g_{A}^{0} \equiv \Delta u+\Delta d+\Delta s$ is small (which is now $0.27 \pm 0.10$ [2] or $0.22 \pm 0.10$ [4]) is now firmly established. Another interesting result is that the strangeness content in the proton, as indicated by $\Delta s=-0.10 \pm 0.04[2]$ or $-0.12 \pm 0.04$ [4], appears to differ significantly from zero.

The proton spin structure function $g_{1}^{p}(x)$ at a given $Q^{2}$ may be specified by

$g_{1}^{p}(x)=\sum_{i} \frac{1}{2} e_{i}^{2} \Delta q_{i}(x)$,

$$
\Delta q_{i}(x) \equiv q_{i}^{+}(x)-q_{i}^{-}(x)+\bar{q}_{i}^{+}(x)-\bar{q}_{i}^{-}(x),
$$

where $q^{ \pm}(x)\left[\bar{q}^{ \pm}(x)\right]$ is the quark (antiquark) distribution with positive helicity or negative helicity, respectively. It is known that the QCD-corrected parton model expression for the integral of $g_{1}^{p}(x)$, i.e. the first moment, is given by

$$
\begin{aligned}
\Gamma_{p}= & \int_{0}^{1} g_{1}^{p}(x) d x=\frac{1}{12}\left\{\left(1-\frac{\alpha_{s}}{\pi}\right)\left(a_{3}+\frac{1}{\sqrt{3}} a_{8}\right)\right. \\
& \left.+2 \sqrt{\frac{2}{3}}\left(1-\frac{33-8 f}{33-2 f} \frac{\alpha_{s}}{\pi} a_{0}\right)\right\},
\end{aligned}
$$

where the quantities $a_{j}$ are directly related to the proton forward matrix elements of the nonet and 
octet of axial vector currents $A_{j}^{\mu}(x)=\bar{\psi}(x) \gamma^{\mu} \gamma_{5^{-}}$ $\left(\lambda_{j} / 2\right) \psi(x), j=0,1, \ldots, 8$ by $\left\langle P, S\left|A_{j}^{\mu}(0)\right| P, S\right\rangle=$ $2 M a_{j} S^{\mu}$ where $S^{\mu}$ is the covariant spin vector of the proton. In the naive parton model, we have

$a_{0}=\sqrt{\frac{2}{3}}\{\Delta u+\Delta d+\Delta s\}$,

$a_{3}=\Delta u-\Delta d$,

$a_{8}=\frac{1}{\sqrt{3}}\{\Delta u+\Delta d-2 \Delta s\}$,

where $\Delta q \equiv \int_{0}^{1} \Delta q(x) d x$.

The axial coupling $g_{A}$, as determined from neutron $\beta$ decay, is [5]

$g_{A} \equiv a_{3}=F+D=1.2601 \pm 0.0025$,

while the quantity $a_{8}$ can be determined from the observed $\beta$ decays of octet baryons,

$a_{8}=\frac{1}{\sqrt{3}}(3 F-D)=0.397 \pm 0.020$.

Accordingly, the flavor-singlet axial coupling $g_{A}^{0}$ $=\sqrt{\frac{3}{2}} a_{0}$ may be determined experimentally from the measured values of $\Gamma_{p}, a_{3}$ and $a_{8}$, leading to what has been cited earlier for the smallness of $g_{A}^{0}$, or, the unexpected smallness of the quark contribution to the proton spin.

There are many possible theoretical interpretations of the proton spin crisis, on which we could only refer the readers to some review article [6]. In this paper, we wish to address a simple way to resolve the proton spin crisis in the context of a quark model, which is to avoid identifying the measured quantities $\Delta u, \Delta d$, and $\Delta s$ as coming exclusively from the valence quark contributions, and which can easily be achieved if we can split $\Delta q$ into the piece from the valence quarks, namely $\Delta q_{\mathrm{val}}$, and another piece from elsewhere, say from the sea, $\Delta q_{\text {sea }}$. As the sum is vanishingly small from the experiment, the valence contribution $\sum \Delta q_{\text {val }}$ has to be cancelled almost completely by the sea contribution $\sum \Delta q_{\text {sea }}$. The introduction of the "sea" complicates the physical picture, a situation which we may want to avoid if possible but which might be the inherent nature of strong interaction physics. Fortu- nately, the present situation is dictated by chiral symmetry breaking in QCD, i.e., breaking of $S U\left(N_{f}\right)_{R} \times S U\left(N_{f}\right)_{L}$ chiral symmetry of QCD onto $S U\left(N_{f}\right)_{V}$ (leaving $N_{f}^{2}-1$ conserved polar vector currents together with $N_{f}^{2}-1$ Goldstone bosons).

The effective chiral quark model $[7,8]$ is a theory describing how the naive constituent quark picture can be modified, upon chiral symmetry breaking, to yield sea quarks (of possibly substantial amount), resulting in a world of quarks, gluons, and Goldstone bosons interacting in an approximately chiral invariant manner. The model may be specified by $[7,8]$

$\mathscr{L}=\bar{\psi}\left(i \mathscr{D}_{\mu}+V_{\mu}\right) \gamma^{\mu} \psi+i g_{A} \bar{\psi} A_{\mu} \gamma^{\mu} \gamma_{5} \psi+\ldots$,

where $\psi$ is a triplet consisting of $u, d$, ans $s$ quarks (each carrying one of three possible colors). $\mathscr{D}_{\mu}=\partial_{\mu}$ $+i g G_{\mu}$ is the gauge-covariant derivative in QCD. Axial-vector coupling $g_{A}(=0.7524)$ is determined from the axial charge of the nucleon. The polar vector and axial vector currents are given by

$\left(\begin{array}{c}V_{\mu} \\ A_{\mu}\end{array}\right)=\frac{1}{2}\left(\xi^{\dagger} \partial_{\mu} \xi \pm \xi \partial_{\mu} \xi^{\dagger}\right)$,

where $\xi=\exp (i \Pi / f)$ with the pion decay constant $f$ of $93 \mathrm{MeV}$ and

$$
\Pi \equiv \frac{1}{\sqrt{2}}\left(\begin{array}{ccc}
\frac{\pi^{0}}{\sqrt{2}}+\frac{\eta}{\sqrt{6}} & \pi^{+} & K^{+} \\
\pi^{-} & -\frac{\pi^{0}}{\sqrt{2}}+\frac{\eta}{\sqrt{6}} & K^{0} \\
K^{-} & \bar{K}^{0} & -\frac{2 \eta}{\sqrt{6}}
\end{array}\right) .
$$

Expansion of the currents in powers of $\Pi / f$ (a chiral perturbation theory) gives rise to an effective interaction between Goldstone bosons and quarks:

$\mathscr{L}_{\text {int. }}=-\frac{g_{A}}{f} \bar{\psi} \gamma^{\mu} \gamma_{5} \psi \partial_{\mu} \Pi$.

In recent years, a number of authors have used the model to analyze different problems. Among them, 
Weinberg [9] used it to calculate the value of $g_{A}$ [9]; Eichten, Hinchliffe and Quigg [10], and recently Cheng and Li [11], used it to show how the $\bar{d}$ over $\bar{u}$ asymmetry within the proton can arise from the dissociation of a quark into a quark plus a Goldstone bosons, and this effect is large enough to account for the observed violation [12] of the Gottfried sum rule [13]. They also show how the picture may offer an explanation on the observed value of the first moment of the proton spin structure function $g_{1}^{p}(x)$.

An important result which we wish to add to the existing literature $[10,11]$ is to determine whether the shape (i.e. as a function of $x$ ) of the observed nucleon spin structure function $g_{1}^{p}(x)$ and $g_{1}^{n}(x)$ can be understood in terms of the same picture. Despite the fact that the integrated value has been obtained (in fact in a relatively easy manner), the location (or the where-about) of the "sea" contribution reflects the origin of the sea and such location can be anywhere depending upon the dynamics.

We may introduce the proton spin structure functions $g_{1}(x)$ and $g_{2}(x)$ by considering the polarized electron-proton deep inelastic scattering (DIS):

$\boldsymbol{e}\left(k_{\mu}\right)+\boldsymbol{p}\left(p_{\mu}\right) \rightarrow e^{\prime}\left(k_{\mu}^{\prime}\right)+X\left(P_{X \mu}\right)$

In the laboratory frame, the momenta of the target proton and of the initial and final electrons are respectively

$p_{\mu}=(M, 0,0,0), \quad k_{\mu}=(E, \boldsymbol{k}), \quad k_{\mu}^{\prime}=\left(E^{\prime}, \boldsymbol{k}^{\prime}\right)$,

and the momentum transfer squared $Q^{2}$ is

$Q^{2} \equiv-q^{2}=-\left(k-k^{\prime}\right)^{2}$

The cross section may be expressed as follows:

$$
\frac{d^{2} \sigma}{d \Omega d E^{\prime}}=\frac{\alpha^{2}}{q^{4}}\left(\frac{E^{\prime}}{E}\right) l^{\mu \nu} W_{\mu \nu},
$$

where $\alpha=e^{2} / 4 \pi, l^{\mu \nu}$ is the leptonic tensor and $W_{\mu \nu}$ the hadronic tensor. We have

$$
W_{\mu \nu} \equiv 4 \pi^{2} \frac{E_{P}}{M} \int d^{4} x e^{i q \cdot x}\left\langle p\left|J_{\mu}^{(h)}(x) J_{\nu}^{(h)}(0)\right| p\right\rangle,
$$

where $J_{\mu}^{(h)}(x)$ is the hadronic electromagnetic current. The antisymmetric part of $W_{\mu \nu}$ may be parametrized as

$$
\begin{gathered}
W_{[\mu \nu]}=i \varepsilon_{\mu \nu \lambda \sigma} q^{\lambda}\left\{S ^ { \sigma } \left[M G_{1}\left(\nu, q^{2}\right)+\frac{p \cdot q}{M} G_{2}\right.\right. \\
\left.\left.\times\left(\nu, q^{2}\right)\right]-S \cdot q p^{\sigma} \frac{G_{2}\left(\nu, q^{2}\right)}{M}\right\}, \\
S=\left(\frac{|\boldsymbol{P}|}{M}, \frac{E_{P}}{M}\left(\frac{\boldsymbol{P}}{|\boldsymbol{P}|}\right)\right) .
\end{gathered}
$$

In the Bjorken limit, we have

$$
\begin{aligned}
& M^{2} \nu G_{1}\left(\nu, q^{2}\right) \rightarrow g_{1}(x), \\
& M \nu^{2} G_{2}\left(\nu, q^{2}\right) \rightarrow g_{2}(x) .
\end{aligned}
$$

To obtain the spin structure functions in the effective chiral quark model, we may follow the formulation developed in great detail by Drell, Levy, and Yan [14], a formulation suitable in the limit of infinite initial hadron momentum (and similar to what we nowadays refer to as the light-cone perturbation theory). In this formalism, the electromagnetic current may be represented as

$$
J_{\mu}(x)=U^{-1}(t) j_{\mu}(x) U(t),
$$

where $j_{\mu}(x)$ may be viewed as the "bare" current, and

$$
\begin{aligned}
& U(t)=T\left(\exp \left[-i \int_{-\infty}^{t} d \tau H_{I}(\tau)\right]\right), \\
& H_{I}(t)=-i \frac{g_{A}}{f}\left(m_{q}+m_{q^{\prime}}\right) \int \bar{\psi}_{q} \Pi_{q q^{\prime}} \gamma_{5} \psi_{q^{\prime}} d^{3} x .
\end{aligned}
$$


Thus, the method allows us to treat the contribution from the cloud of Goldstone bosons as the term to first order in $H_{I}(t)$. Further manipulations of Eqs. (18) and (17) in the context of Eq. (14) lead to a number of "light-cone Feynman rules" for computing the various amplitudes such as Eq. (14). To save space, we need to leave out the details of the derivation $[15,14]$ and just quote the final expressions:

$$
\begin{aligned}
g_{1}(x)= & \sum_{i} e_{i}^{2} \Delta q_{i}(x), \quad g_{2}(x)=0, \\
\Delta q_{i}(x)= & Z_{i} \Delta q^{0}(x) \\
& \quad+\sum_{j, C} \int_{0}^{1} d y \frac{1}{y} \Delta \mathscr{P}_{j \rightarrow i C}(y) \Delta q_{j}^{0}(x / y) .
\end{aligned}
$$

Here the $Z$-factor is given by

$$
\begin{aligned}
Z= & 1-\frac{1}{16 \pi^{2}} g_{A \rightarrow B C}^{2} \int_{0}^{1} d y \int_{0}^{k_{\perp \max }^{2}(y)} d k_{\perp}^{2} \\
& \times \frac{(1-y)\left(k_{\perp}^{2}+\left(m_{B}-y m_{A}\right)^{2}\right)}{\left(k_{\perp}^{2}-y(1-y) m_{A}^{2}+(1-y) m_{B}^{2}+y m_{C}^{2}\right)^{2}} \\
& \times \Theta\left(y-y_{0}\right) \\
\equiv & 1-\int_{0}^{1} d y \mathscr{P}_{A \rightarrow B C}(y) .
\end{aligned}
$$

with $\mathscr{P}_{A \rightarrow B C}(y)$ the splitting function for $A \rightarrow B C$, with $y$ and $1-y$ the momentum fractions of $B$ and $C$. Note that the integration range of $d k_{\perp}^{2} d y$ is given by

$0 \leq k_{\perp}^{2} \leq k_{\perp \max }^{2}(y), \quad y_{0} \leq y \leq 1$,

where

$k_{\perp \max }^{2}(y)=y \Lambda^{2}-m_{B}^{2}(1-y)+m_{A}^{2} y(1-y)$,

and $y_{0}$ satisfies $k_{\perp \max }^{2}\left(y_{0}\right)=0$. This is in turn related to the assumption that the effective chiral quark model is valid up to certain high energy:

$t \equiv\left(p_{A}-p_{B}\right)^{2}>-\Lambda^{2}, \quad \Lambda=\Lambda_{\chi S B} \equiv 4 \pi f$.

We shall treat $\Lambda$ as an adjustable parameter (fixed at a value of about $3 \mathrm{GeV}$ ) in obtaining numerical predictions.
In the naive quark model, the valence distributions are given by "bare" contribution (the zerothorder result):

$u^{+}(x)=\frac{5}{3}\left(u_{v}(x) / 2\right), \quad u^{-}(x)=\frac{1}{3}\left(u_{v}(x) / 2\right) ;$

$d^{+}(x)=\frac{1}{3}\left(d_{v}(x)\right), \quad d^{-}(x)=\frac{2}{3}\left(d_{v}(x)\right) ;$

$q^{+}(x)+q^{-}(x)=q_{v}(x)$,

which yields

$\Delta u^{0}(x)=\frac{4}{3}\left(u_{v}(x) / 2\right), \quad \Delta d^{0}(x)=-\frac{1}{3} d_{v}(x)$.

However, this input would be inconsistent with the data [6] which indicates $A_{1}^{p}(x) \equiv g_{1}(x) / F_{1}(x) \rightarrow 1$ as $x \rightarrow 1$. To ensure this result in employing the formulae (19)-(22), we may choose, keeping in mind that $d_{v}(x) / u_{v}(x) \rightarrow 0$ as $x \rightarrow 1$,

$\Delta u^{0}(x)=\frac{4}{3}\left\{\frac{3}{4}\left(u_{v}(x) / 2\right)+\frac{1}{4} d_{v}(x)\right\}$,

$\Delta d^{0}(x)=-\frac{1}{3} d_{v}(x)$.

Note that $\int \Delta u^{0}(x) d x=\frac{4}{3}$ remains intact. To obtain numerical results, we adopt the parametrization of Glück, Reya, and Vogt [16] for $u_{v}\left(x, Q^{2}\right)$ and $d_{v}\left(x, Q^{2}\right)$. Such choice does not affect much our final results since the valence quark distributions do not vary significantly with the various parametrizations. All of the physical constants, such as masses of quarks, are from EHQ and Georgi $[8,10]$.

Although the chiral quark model can in principle be used to predict the valence distributions of the proton, such task is yet to be clearly formulated but it is clear that the commonly-assumed parton behavior near $x \rightarrow 0$ (the so-called "Reggie behavior") can hardly be understood directly from the valence quark model. Accordingly, we choose to resort to the experiments (which are used to obtain the fitted parton distributions) for obtaining the input on the valence distributions (of the proton and the Goldstone pions). The calculations to obtain the sea distributions can be performed first at a $Q^{2}$ (e.g., in the vicinity of (1-2) $\mathrm{GeV}^{2}$ ) which the chiral quark model is presumably valid, and the profile of the entire parton distributions can then be evolved to some desirable higher $Q^{2}$.

In this way, we obtain, at $Q^{2}=10 \mathrm{GeV}^{2}$, $\Delta u=0.705, \quad \Delta d=-0.307, \quad \Delta s=-0.074$, 

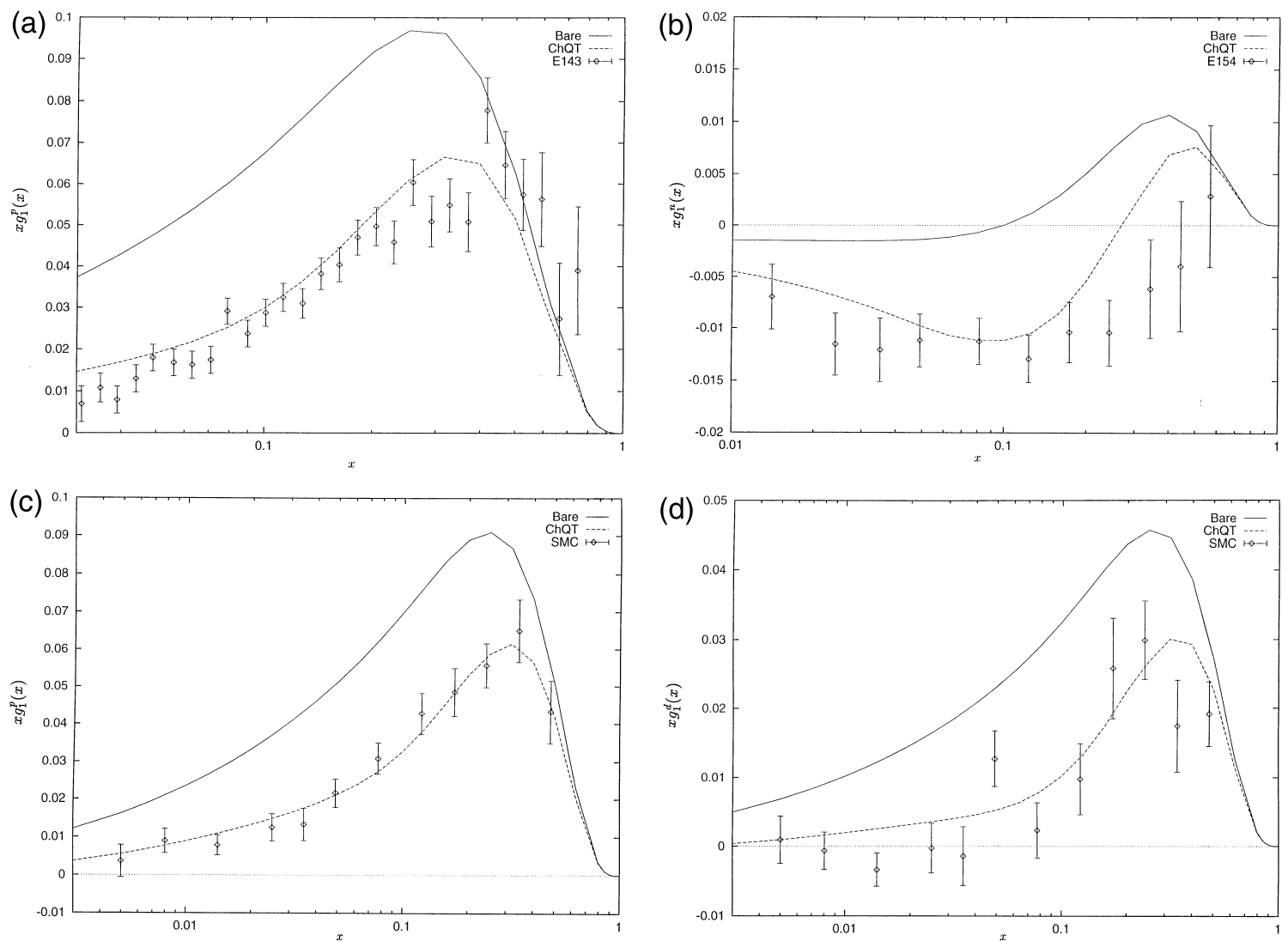

Fig. 1. (a)-(d): The spin structure functions $x g_{1}^{p}(x)$ and $x g_{1}^{n}(x)$ [and $x g_{1}^{d}(x)$ for 1(d)] are shown as functions of the Bjorken scaling variable $x$ at suitable $Q^{2}$. The solid curve, as denoted as "Bare", represents the prediction to zeroth order, i.e., without the meson cloud. The dashed curve, as labeled by "ChQT", is our full prediction (i.e., to first order, or with the meson cloud). The data are from [2-4].

which are to be compared with those extracted from the EMC data [1]:

$\Delta u=0.780 \pm 0.032 \pm 0.046$

$\Delta d=-0.472 \pm 0.032 \pm 0.046$,

$\Delta s=-0.190 \pm 0.032 \pm 0.046$.

The first moment of $g_{1}^{p}(x)$ may also be obtained:

$$
\begin{aligned}
\Gamma_{p}= & \int_{0}^{1} g_{1}^{p} d x=\frac{1}{2}\left(\frac{3.82}{9} \Delta u+\frac{1.08}{9} \Delta d\right. \\
& \left.+\frac{1.08}{9} \Delta s\right)=0.135,
\end{aligned}
$$

and, analogously,

$$
\Gamma_{n}=-0.033, \quad \Gamma_{p}-\Gamma_{n}=0.168 .
$$

These values compare very well with the E154 data [3] of $\Gamma^{n}=-0.041 \pm 0.007$ and $\Gamma_{p}-\gamma_{n}=0.171 \pm$ 0.013. They are consistent with, albeit slightly lower (in magnitude) than, the E143 and SMC data, as well as a theoretical treatment [17] of the Bjorken sum rule [18] $\left(\Gamma_{p}-\Gamma_{n}=0.186 \pm 0.003\right)$.

We turn our attention to the functional form (the shape) of the spin structure functions $g_{1}^{p}(x)$ and $g_{1}^{n}(x)$, as displayed in Fig. 1(a)-(d). The solid curve, as denoted as "Bare", represents the prediction to zeroth order, i.e., without the meson cloud. The dashed curve, as labeled by "ChQT', is our full 
prediction (i.e., to first order, or with the meson cloud), which agrees very well with the E154, E143, and SMC data, respectively.

We wish to emphasize that the effects due to the meson cloud are treated, with greater complication, at the hadronic level $[19,20]$, resulting in predictions which are similar to what we have reported in this paper. It is clear that the nonperturbative effects played by Goldstone bosons offer the most natural explanation. It seems unlikely that an explanation without Goldstone bosons could produce the observed shape in a straightforward manner.

In summary, we have shown how the nucleon spin structure functions, as extracted from the recent experiments carried out by the European Muon Collaboration (EMC) [1], the SLAC E143 and E154 Collaborations [2,3], and the Spin Muon Collaboration (SMC) [4], can be understood quantitatively in the context of the effective chiral quark model, both for the measured first moments $\Gamma_{1} \equiv \int_{0}^{1} g_{1}(x) d x$ and for the functional form (or the shape) of the spin structure function $g_{1}(x)$ (as a function of the Bjorken scaling variable $x$ ). The success strongly suggests that the nonperturbative effects due to Goldstone bosons play a significant role, and should be taken into account, in the kinematic range of the momentum transfer squared $Q^{2}$ of several $\mathrm{GeV}^{2}$.

\section{Acknowledgements}

This work was supported in part by the National Science Council of R.O.C. (NSC87-2112-M002017Y and NSC88-2112-M002-001Y).

\section{References}

[1] European Muon Collaboration (EMC), J. Ashman et al., Phys. Lett. B 206 (1988) 1167; J. Ashman et al., Nucl. Phys. B 328 (1989) 1.

[2] E143 Collaboration, K. Abe et al., Phys. Rev. Lett. 74 (1995) 346; Phys. Lett. B 405 (1997) 180.

[3] E154 Collaboration, K. Abe, Phys. Rev. Lett. 79 (1997) 26.

[4] SMC Collaboration, D. Adame et al., Phys. Lett. B 329 (1994) 399; B. Adeva et al., Phys. Lett. B 412 (1997) 414; B 396 (1997) 338.

[5] C. Caso et al., Particle Data Group, Review of Particle Physics, Eur. Phys. J. C 3 (1998) 1

[6] For a review, see M. Anselmino, A. Efremov, E. Leader, Phys. Rep. 261 (1995) 1.

[7] S. Weinberg, Phys. Lett. B 251 (1990) 288; Physics 96A (1979) 327

[8] H. Georgi, Weak interaction and modern particle theory, Benjamin /Cummings Publishing Co., Menlo Park, CA, 1984; A. Manohar, H. Georgi, Nucl. Phys. B 234 (1984) 189.

[9] S. Weinberg, Phys. Rev. Lett. 67 (1991) 3474.

[10] E.J. Eichten, I. Hinchliffe, C. Quigg, Phys. Rev. D 45 (1992) 2269.

[11] T.P. Cheng, Ling-Fong Li, Phys. Rev. Lett. 74 (1995) 2872.

[12] New Muon Collaboration, P. Amaudruz et al., Phys. Rev. Lett. 66 (1991) 2712; M. Arneodo et al., Phys. Rev. D 50 (1994) R1.

[13] K. Gottfried, Phys. Rev. 18 (1967) 1174.

[14] S.D. Drell, D.J. Levy, T.-M. Yan, Phys. Rev. D 1 (1970) 1035.

[15] C.-K. Chua, W-Y.P. Hwang, Chinese J. Physics (Taipei) 36 (1998) 588; C.-K. Chua, M.S. Thesis, National Taiwan University, 1994.

[16] M. Glück, E. Reya, A. Vogt, Z. Phys. C 67 (1995) 433.

[17] S.A. Larin, Phys. Lett. B 334 (1994) 192.

[18] J.D. Bjorken, Phys. Rev. 148 (1966) 1467.

[19] W-Y.P. Hwang, J. Speth, G.E. Brown, Z. Phys. A 339 (1991) 383.

[20] C.-H. Chung, W-Y.P. Hwang, Phys. Rev. 49 (1994) 2221; J. Speth, private communication. 